\title{
Tomografia computerizzata delle coronarie: piccolo vademecum per il nefrologo
}

\author{
Fulvio Floccari ${ }^{1}$, Rodolfo Rivera ${ }^{2}$, Luca Di Lullo ${ }^{3}$ \\ ${ }^{1}$ U.O.C. Nefrologia e Dialisi, Ospedale S. Paolo, Civitavecchia (RM) \\ ${ }^{2}$ Divisione di Nefrologia, Ospedale S. Gerardo, Monza \\ ${ }^{3}$ U.O.C. Nefrologia e Dialisi, Ospedale L. Parodi-Delfino, Colleferro (RM)
}

CORONARY COMPUTED TOMOGRAPHY: A NEW TOOL FOR NEPHROLOGIST

Abstract. Coronary computed tomography (CT) angiography has been widely employed due to the better spatial and temporal resolution.

The diagnostic value of coronary CT is gradually improved together with the evolution of multislice CT scans from the original 4-slice scan to the actual 320-slice scan.

Despite its diagnostic and prognostic value, the use of coronary CT angiography is actually limited to restricted fields due to the high prevalence of artifacts and elevated number of false-positive results related to the high density of the valvular and vascular calcifications.

Aim of this review is to present the technological development of coronary CT during the last decades, together with its prognostic involvement.

Key words: Coronary CT scan angiography, Multislice, Coronary disease, Valvular calcifications, Vascular calcifications

Conflict of interest: None.

Financial support: None.

Accettato: 29 Maggio 2014

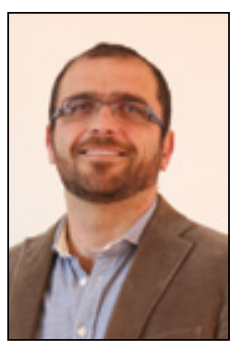

Floccari Fulvio

\section{Introduzione}

La tomografia computerizzata delle coronarie, o cardio-TC, sta trovando un utilizzo sempre più diffuso nella diagnosi dell'arteriopatia coronarica, grazie alla non invasività della metodica, all'elevata risoluzione spazio-temporale e al ridotto utilizzo di mezzo di contrasto.

Tale metodica riguarda da vicino il nefrologo, che potrebbe talvolta essere nelle condizioni di proporne l'utilizzo al collega cardiologo. È infatti da sottolineare come la coronarografia tradizionale preveda l'infusione intra-arteriosa di mezzo di contrasto, correlata maggiormente alla nefropatia da contrasto rispetto alla semplice infusione endovenosa (1) della cardio-TC. Obiettivo di questa breve dissertazione in materia sarà, quindi, di presentare brevemente al nefrologo le innovazioni tecnologiche più recenti e di valutare le evidenze di letteratura circa le performance diagnostiche della cardio-TC.

La capacità diagnostica di questa metodica è andata progressivamente crescendo negli anni, insieme alla risoluzione spaziotemporale, tanto maggiore quanto più elevato è il numero di strati di tessuto analizzati con una singola rotazione del gantry.
Si è passati infatti dai primi esemplari a 4 strati del 1998 a quelli a 16 del 2002, per arrivare ai 64 del 2008 e, così, agli ultimi avveniristici modelli da 128, 256 o, addirittura, 320 strati (2-4).

\section{L'importanza del numero degli strati}

Il ciclo cardiaco e quello respiratorio condizionano pesantemente l'esecuzione di un esame cardio-TC, con una grande quantità di artefatti da movimento. Al concetto di definizione spaziale dell'esame deve essere infatti affiancato quello di definizione temporale (5).

La capacità del paziente di mantenere l'apnea è utile a minimizzare gli effetti artefattuali dovuti ai movimenti del torace, mentre escludere dall'esame i movimenti intrinseci alla meccanica cardiaca richiede che l'acquisizione delle immagini sia estremamente rapida (5).

La TC multislice consente la lettura di un singolo fotone da parte di un numero elevato di sensori (proporzionale al numero di strati analizzabili) in pochi secondi, con durate medie di un esame cardio-TC che sono così passate da 50 secondi circa a 15 secondi (6).

Le apparecchiature più moderne hanno ulteriormente ridotto tali tempi, ricorrendo a due sorgenti emissive e a due unità 
indipendenti di sensori. Tale innovazione ha portato i tempi di rotazione del gantry a 280 millisecondi ( 0.28 secondi!). La presenza di due sorgenti consente inoltre di acquisire due fotoni per ogni sensore, facendo sì che una $\mathrm{TC}$ a 64 sensori ottenga immagini a 128 strati $(7,8)$.

Utilizzando le due sorgenti con voltaggi tra loro differenti (Dual Energy C, DECT), è possibile aumentare la capacità di differenziazione tissutale, portando l'accuratezza diagnostica per l'arteriopatia coronarica a oltre il $90 \%(9,10)$.

Lo strumento a maggiore capacità di copertura dell'asse $\mathrm{z}$ è l'Aquilion One Dynamic Volume della Toshiba, con una capacità di scansione di 320 strati e la conseguente peculiare capacità di acquisizione di tutte le immagini in un solo ciclo cardiaco. Recenti metanalisi dimostrerebbero un valore predittivo negativo vicino al $100 \%$ per apparecchiature di tale livello tecnologico (11-13).

Oltre ad agire sulla rapidità di acquisizione delle immagini, i produttori di apparecchiature radiologiche hanno affinato anche la capacità degli strumenti di sincronizzare l'esame al ciclo cardiaco mediante l'acquisizione di un elettrocardiogramma $(14,15)$. Tale capacità è passata così da sistemi che effettuavano la sincronizzazione in modalità retrospettiva (quindi, operando in post-processing la sovrapposizione tra ECG e immagini una volta che entrambi sono stati acquisiti) a sistemi che invece acquisiscono le immagini sincronizzandole in modalità prospettica con l'ECG in acquisizione. Tale innovazione ha ulteriormente incrementato il valore predittivo negativo della metodica, portandolo a un valore compreso tra il $96 \%$ e il $99 \%$. Normalmente, un'apparecchiatura a sincronia-ECG prospettica a 64-128 strati riesce a completare un esame cardio-TC in soli tre battiti cardiaci, utilizzando il primo ciclo cardiaco per la metà superiore del cuore, il secondo per traslare verso i ventricoli e il terzo per scansionarli $(16,17)$.

\section{I limiti della tecnologia}

La presenza di frequenze cardiache superiori ai $65 \mathrm{bpm}$ o di irregolarità del ritmo cardiaco, dovute a extrasistoli o a fibrillazione atriale comporta la presenza di artefatti da movimento nelle immagini ricostruite. Molti costruttori hanno cercato di affrontare tale problematica con appositi software che effettuano la ricostruzione delle immagini acquisite nella seconda metà della diastole, normalmente meno affetta da extrasistoli.

Per ridurre il rischio di artefatti di tale tipologia, una dose massima di $15 \mathrm{mg}$ di propanololo o di $40 \mathrm{mg}$ di diltiazem è comunemente utilizzata come preparazione all'esame $(18,19)$.

In presenza di controindicazioni assolute all'utilizzo di Bbloccanti o di diltiazem, l'unica strategia in grado di minimizzare la presenza di artefatti da movimento è l'utilizzo di un'apparecchiatura capace di un'acquisizione ECG-prospettica delle immagini, a elevato numero di strati. Apparecchiature prospettiche a 320 strati sembrano infatti essere completamente indipendenti dalla frequenza cardiaca nella qualità delle immagini prodotte (20).

Come detto, la risoluzione temporale è uno dei principali limiti tecnici della cardio-TC. Le apparecchiature a sorgente singola affrontano questa problematica riducendo il pitch, ossia riducendo la velocità di traslazione dei sensori, al costo di un corposo incremento della dose radiante. Apparecchiature DECT ECG-prospettiche migliorano grandemente tale aspetto, ottenendo risoluzioni temporali di 75 millisecondi, comunque elevate rispetto ai $10 \mathrm{~ms}$ di una coronarografia tradizionale. La riduzione della dose radiante, già notevolmente ridotta in apparecchiature a 128-slice o superiori, è ottenuta inoltre attraverso l'utilizzo di collimatori dinamici elicoidali e adattativi assiali, che ottimizzano le scansioni e la durata dell'esame $(21,22)$.

La presenza di calcificazioni severe condiziona ulteriormente la qualità delle immagini. I depositi di calcio hanno un'attenuazione radiologica talmente elevata da attenuare notevolmente i raggi $\mathrm{X}$ a bassa energia utilizzati dalle apparecchiature $\mathrm{TC}(23,24)$. L'utilizzo di radiazioni a maggiore energia comporta una perdita di definizione, solo in parte compensata da apparecchiature con $>64$ strati grazie alla riduzione delle dimensioni dei voxel, con il rischio di sovrastimare l'entità delle placche che si aggiunge all'incremento della dose radiante (25). Evidenze in letteratura dimostrano come un Agatston score superiore a 400 rappresenti ancora oggi un limite tecnico difficilmente valicabile per un'adeguata qualità delle immagini con le apparecchiature più diffuse. I dati ottenuti al riguardo con apparecchiature a 256 o a 320 strati risultano ancora numericamente insufficienti per valutare se la performance diagnostica di TC di ultima generazione sia affidabile anche in questa particolare fattispecie di pazienti, così comune nella casistica nefrologica (26).

\section{Conclusioni}

La capacità diagnostica della cardio-TC, specialmente in modalità DECT, nella visualizzazione e nella caratterizzazione delle placche è notevole.

Diversi studi ne hanno riportato la capacità predittiva nei confronti degli eventi cardiaci avversi in pazienti con CAD nota o sospetta (27-30). Ostrom et al. e Min et al. $(31,32)$ hanno riportato una correlazione diretta tra mortalità e numero di vasi interessati da placche sia ostruttive che non ostruttive.

L'utilizzo di questa metodica in pazienti asintomatici, come alternativa alla coronarografia tradizionale squisitamente diagnostica, offre, poi, indubbi vantaggi di natura economica anche davanti a un'affidabilità diagnostica di tutto rispetto (33).

L'esame è poi decisamente meno problematico per il paziente sotto il profilo umano, risultando assolutamente non invasivo ed essendo erogabile in modalità ambulatoriale.

Sulla base di quanto sopra riportato, la cardio-TC può, quindi, aprire nuovamente l'iter diagnostico della CAD in pazienti che risultano non eleggibili alla coronarografia classica?

Non abbiamo risposte a questa domanda. Certamente, ancora una volta, si chiede al nefrologo clinico di allargare la propria sfera delle conoscenze, per aggiungere questa nuova metodica al carnet delle proprie opzioni diagnostiche e per interagire con consapevolezza con i colleghi cardiologi. 


\section{Riassunto}

La tomografia (TC) coronarica viene utilizzata su larga scala grazie alle sua caratteristiche di migliore risoluzione spazio-temporale.

Il valore diagnostico della $\mathrm{TC}$ coronarica è andato via via migliorando grazie alla sua evoluzione in TC multistrato; si è, infatti, passati dagli originari 4 strati agli attuali 320 strati, con un netto guadagno in termini di risoluzione e, quindi, di nitidezza delle immagini.

Nonostante l'indubbio valore diagnostico e, in parte, anche prognostico, la TC coronarica presenta indicazioni limitate in alcuni specifici campi di applicazione, in virtù dell'elevata prevalenza di artefatti e di falsi positivi determinati dalla presenza di calcificazioni vascolari e/o valvolari. Scopo della presente rassegna è quello di presentare l'evoluzione tecnologica della TC coronarica negli ultimi anni e le sue implicazioni dal punto di vista prognostico.
Parole chiave: TC coronarica, Multistrato, Malattia coronarica, Calcificazioni valvolari, Calcificazioni vascolari

Dichiarazione di conflitto di interessi: Gli Autori dichiarano di non avere conflitto di interessi.

Contributi economici agli Autori: Gli Autori dichiarano di non aver ricevuto sponsorizzazioni economiche per la preparazione dell'articolo.

\section{Indirizzo degli Autori:}

Dr. Fulvio Floccari

U.O.C. Nefrologia e Dialisi

Ospedale S. Paolo

Largo Donatori di Sangue 1

00053 Civitavecchia (RM)

fulviofloccari@gmail.com

\section{Bibliografia}

1. Patel MR, Bailey SR, Bonow RO, et al. ACCF/SCAI/AATS/ AHA/ASE/ASNC/HFSA/HRS/SCCM/SCCT/SCMR/STS 2012 appropriate use criteria for diagnostic catheterization: a report of the American College of Cardiology Foundation Appropriate Use Criteria Task Force, Society for Cardiovascular Angiography and Interventions, American Association for Thoracic Surgery, American Heart Association, American Society of Echocardiography, American Society of Nuclear Cardiology, Heart Failure Society of America, Heart Rhythm Society, Society of Critical Care Medicine, Society of Cardiovascular Computed Tomography, Society for Cardiovascular Magnetic Resonance, Society of Thoracic Surgeons. J Thorac Cardiovasc Surg 2012; 144 (1): 39-71.

2. Paul JF, Dambrin G, Caussin C, Lancelin B, Angel C. Sixteenslice computed tomography after acute myocardial infarction: from perfusion defect to the culprit lesion. Circulation 2003; 108: 373-4.

3. Ropers D, Baum U, Pohle K, et al. Detection of coronary artery stenoses with thin-slice multi-detector row spiral computed tomography and multiplanar reconstruction. Circulation 2003; 107: 664-6.

4. Sun Z, Choo GH, Ng KH. Coronary CT angiography: current status and continuing challenges. Br J Radiol 2012; 85: 495-510.

5. Barrett JF, Keat N. Artifacts in CT: recognition and avoidance. Radiographics 2004; 24: 1679-91.

6. Taguchi K, Aradate H. Algorithm for image reconstruction in multi-slice helical CT. Med Phys 1998; 25: 550-61.

7. Flohr TG, Schaller S, Stierstorfer K, Bruder H, Ohnesorge BM, Schoepf UJ. Multi-detector row CT systems and imagereconstruction techniques. Radiology 2005; 235: 756-73.

8. McCollough CH, Zink FE. Performance evaluation of a multislice CT system. Med Phys 1999; 26: 2223-30.
9. Ruzsics B, Lee H, Zwerner PL, Gebregziabher M, Costello P, Schoepf UJ. Dual-energy CT of the heart for diagnosing coronary artery stenosis and myocardial ischemia-initial experience. Eur Radiol 2008; 18: 2414-24.

10. Schwarz F, Ruzsics B, Schoepf UJ, et al. Dual-energy CT of the heart-principles and protocols. Eur J Radiol 2008; 68: 423-33.

11. Mori S, Endo M, Obata T, et al. Clinical potentials of the prototype 256-detector row CT-scanner. Acad Radiol 2005; 12: 148-54.

12. Mori S, Endo M, Obata T, Tsunoo T, Susumu K, Tanada S. Properties of the prototype 256-row (cone beam) CT scanner. Eur Radiol 2006; 16: 2100-8.

13. Mori S, Kondo C, Suzuki N, et al. Volumetric cine imaging for cardiovascular circulation using prototype 256-detector row computed tomography scanner (4-dimensional computed tomography): a preliminary study with a porcine model. J Comput Assist Tomogr 2005; 29: 26-30.

14. Sun Z, Jiang W. Diagnostic value of multislice computed tomography angiography in coronary artery disease: a metaanalysis. Eur J Radiol 2006; 60: 279-86.

15. Pontone G, Andreini D, Bartorelli AL, et al. Diagnostic accuracy of coronary computed tomography angiography: a comparison between prospective and retrospective electrocardiogram triggering. J Am Coll Cardiol 2009; 54: 346-55.

16. van Velzen JE, de Graaf FR, Kroft LJ, et al. Performance and efficacy of 320-row computed tomography coronary angiography in patients presenting with acute chest pain: results from a clinical registry. Int J Cardiovasc Imaging 2012; 28 : 865-76.

17. Pelliccia F, Pasceri V, Evangelista A, et al. Diagnostic accuracy of 320-row computed tomography as compared with invasive coronary angiography in unselected, consecutive patients with suspected coronary artery disease. Int J Cardiovasc Imaging 2013; 29: 443-52. 
18. Pannu HK, Alvarez W, Fishman EK. Beta-blockers for cardiac CT: a primer for the radiologist. AJR Am J Roentgenol 2006; 186: S341-5.

19. Pannu HK, Sullivan C, Lai S, Fishman EK. Evaluation of the effectiveness of oral Beta-blockade in patients for coronary computed tomographic angiography. J Comput Assist Tomogr 2008; 32: 247-51.

20. Hoe J, Toh KH. First experience with 320-row multidetector CT coronary angiography scanning with prospective electrocardiogram gating to reduce radiation dose. J Cardiovasc Comput Tomogr 2009; 3: 257-61.

21. Ketelsen D, Thomas C, Werner M, et al. Dual-source computed tomography: estimation of radiation exposure of ECGgated and ECG-triggered coronary angiography. Eur J Radiol 2010; 73: 274-9.

22. Dikkers R, Greuter MJ, Kristanto W, et al. Assessment of image quality of 64-row Dual Source versus Single Source CT coronary angiography on heart rate: a phantom study. Eur $\mathrm{J}$ Radiol 2009; 70: 61-8.

23. Brodoefel H, Burgstahler C, Tsiflikas I, et al. Dual-source CT: effect of heart rate, heart rate variability, and calcification on image quality and diagnostic accuracy. Radiology 2008; 247: 346-55.

24. Diederichsen AC, Petersen H, Jensen LO, et al. Diagnostic value of cardiac 64-slice computed tomography: importance of coronary calcium. Scand Cardiovasc J 2009; 43: 337-44.

25. Stolzmann P, Scheffel H, Leschka S, et al. Influence of calcifications on diagnostic accuracy of coronary CT angiography using prospective ECG triggering. AJR Am J Roentgenol 2008; 191: 1684-9.

26. Chen $\mathrm{CC}$, Chen $\mathrm{CC}$, Hsieh IC, et al. The effect of calcium score on the diagnostic accuracy of coronary computed tomography angiography. Int J Cardiovasc Imaging 2011; 27 (Suppl. 1): 37-42.

27. Gaemperli O, Valenta I, Schepis T, et al. Coronary 64-slice CT angiography predicts outcome in patients with known or suspected coronary artery disease. Eur Radiol 2008; 18: 1162-73.

28. Carrigan TP, Nair D, Schoenhagen P, et al. Prognostic utility of 64-slice computed tomography in patients with suspected but no documented coronary artery disease. Eur Heart J 2009; 30: 362-71.

29. Chow BJ, Wells GA, Chen L, et al. Prognostic value of 64-slice cardiac computed tomography severity of coronary artery disease, coronary atherosclerosis, and left ventricular ejection fraction. J Am Coll Cardiol 2010; 55: 1017-28.

30. Miszalski-Jamka T, Klimeczek P, Banyś R, et al. The composition and extent of coronary artery plaque detected by multislice computed tomographic angiography provides incremental prognostic value in patients with suspected coronary artery disease. Int J Cardiovasc Imaging 2012; 28: 621-31.

31. Ostrom MP, Gopal A, Ahmadi N, et al. Mortality incidence and the severity of coronary atherosclerosis assessed by computed tomography angiography. J Am Coll Cardiol 2008; 52 (16): 1335-43.

32. Min JK, Feignoux J, Treutenaere J, Laperche T, Sablayrolles J. The prognostic value of multidetector coronary CT angiography for the prediction of major adverse cardiovascular events: a multicenter observational cohort study. Int J Cardiovasc Imaging 2010; 26 (6): 721-8.

33. Schlett CL, Banerji D, Siegel E, et al. Prognostic value of CT angiography for major adverse cardiac events in patients with acute chest pain from the emergency department: 2-year outcomes of the ROMICAT trial. JACC Cardiovasc Imaging 2011; 4: 481-91. 